\title{
Particle Image Velocimetry Measurements of a Dry Powder Inhaler Flow
}

\author{
V. Chaugule ${ }^{1 *}$, L. G. dos Reis ${ }^{2}$, D. F. Fletcher ${ }^{3}$, P. M. Young ${ }^{2,4}$, D. Traini ${ }^{2,5}$, J. Soria ${ }^{1}$ \\ ${ }^{1}$ Laboratory for Turbulence Research in Aerospace and Combustion (LTRAC), Department of Mechanical and Aerospace \\ Engineering, Monash University, Clayton, Victoria 3800, Australia \\ 2 Respiratory Technology, Woolcock Institute of Medical Research, NSW 2037 Sydney, Australia \\ ${ }^{3}$ School of Chemical and Biomolecular Engineering, The University of Sydney, Sydney, Australia \\ ${ }^{4}$ Department of Marketing, Macquarie Business School, Macquarie University, NSW 2109, Australia \\ ${ }^{5}$ Department of Biomedical Sciences, Faculty of Medicine, Health and Human Sciences, Macquarie University, NSW 2109, \\ Australia \\ *vishal.chaugule@monash.edu
}

\begin{abstract}
Inhalation therapy for respiratory disorders is being increasingly delivered via dry powder inhalers (DPIs), which are breath-actuated devices that deliver pharmaceutical drug particles to the lungs. The motion of inhalation air, produced when a patient inhales through this device, supplies all energy for the entrainment, de-agglomeration, and dispersion of powder drug agglomerates into a fine drug particle aerosol. The aerosol performance is directly related to the fluid-mechanics of a given DPI device. These flow mechanisms are complex as they depend on the device design, inhalation flow rate, and the properties of the dry powder formulation used. Among these, the role of device design is crucial as it significantly affects not only the generation and properties of delivered aerosol, but also the capability of targeted regional drug deposition.
\end{abstract}

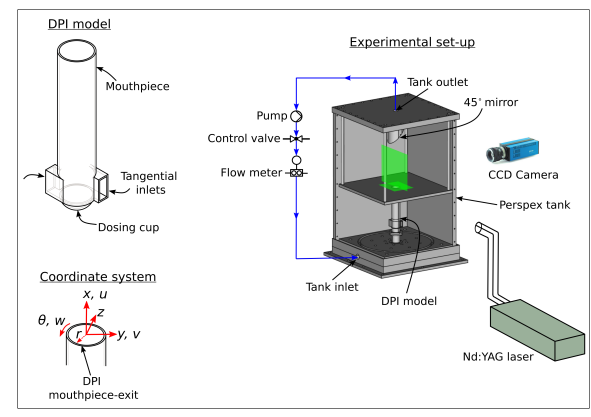

Figure 1: DPI model examined and schematic of the experimental set-up

A common design feature in several DPI devices is the provision of tangential inlets to generate a swirling inhalation flow for the entrainment and de-agglomeration of the drug powder. A typical DPI model based on this design is shown in Fig. 1. It is a hollow circular pipe with two diametrically opposite tangential inlets placed above a hemispherical drug-dosing cup at the bottom, while the open top forms the device mouthpiece. Two component-two dimensional (2C-2D) PIV measurements have been performed on this model using water-based experiments under geometrically and dynamically similar conditions to actual DPIs operating in air (dos Reis et al., 2021). A schematic of the experimental set-up is shown in Fig. 1. The DPI model is placed in the bottom of a tank, both of which are made of clear Perspex, with a closed-loop steady water flow-rate of $12 \mathrm{~L} / \mathrm{min}$ maintained through the system. PIV measurements are taken in longitudinal and transverse planes, both inside and outside the DPI mouthpiece. The transverse plane measurements are taken by imaging the CCD camera through a $45^{\circ}$ mirror mounted inside the tank onto the observation plane defined by the laser sheet. The PIV images are analyzed using the multi-grid/multi-pass cross-correlation digital particle image velocimetry (MCCDPIV) introduced by Soria (1996). The coordinate system adopted is also shown in Fig.11, with its origin just outside the centre of the DPI mouthpiece-exit. The flow $\operatorname{Re}=$ 8400 is defined based on the mouthpiece-exit inner diameter $D=30 \mathrm{~mm}$ and the average axial-exit flow velocity $U_{w}$.

The mean axial $U$, lateral $V$, and tangential $W$ velocity profiles inside and outside the DPI mouthpiece are shown in Fig.2 (a), (b) and (c), respectively. The mean axial velocities in the central flow region become 
increasingly negative as we move from inside to the outside of the mouthpiece. The regions around the mouthpiece edges, $y / D= \pm 0.5$, have high positive mean axial velocities, accompanied by high mean lateral velocities. These characteristics are attributes of a strongly swirling pipe flow inside the DPI mouthpiece which transforms into a swirling jet emerging from it. The mean tangential velocities inside the DPI at $x / D=-3$ attain a maximum value of about $2.7 U_{w}$, which shows the strongly swirling nature of the internal flow, and the swirl level reduces as we move towards the mouthpiece-exit.
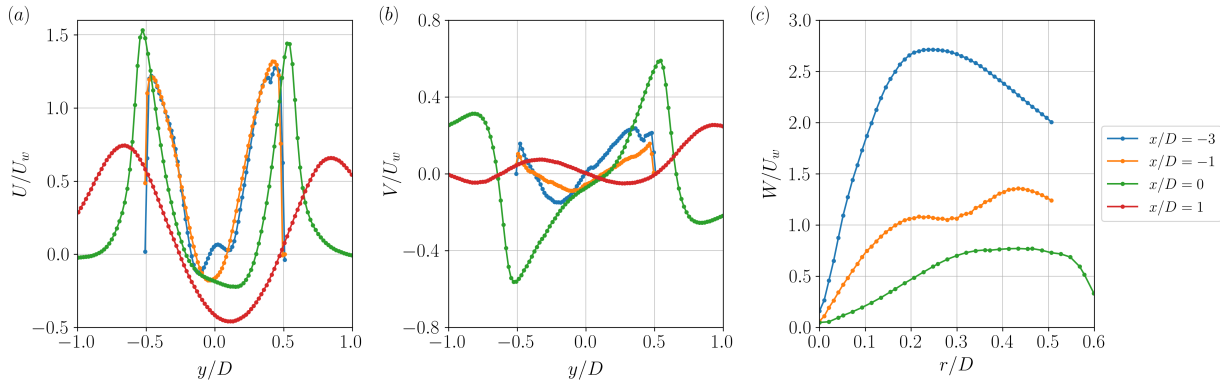

Figure 2: Mean velocities inside and outside the DPI mouthpiece in: (a) axial; (b) lateral; (c) tangential directions.
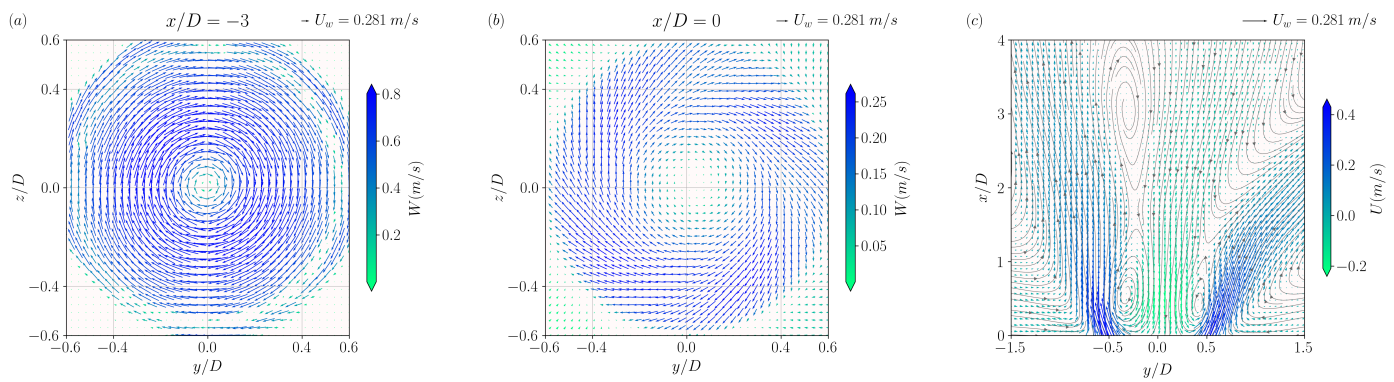

Figure 3: Flow-field vectors on transverse planes inside the DPI mouthpiece at: (a) $x / D=-3$; outside at (b) $x / D=0$; and on a (c) longitudinal plane outside the DPI mouthpiece.

The velocity vectors in the transverse planes $(y-z)$ inside the DPI mouthpiece, at $x / D=-3$, and outside, at $x / D=0$, are illustrated in Fig. 3 (a) and (b), respectively, while those in the longitudinal plane $(x-y)$ outside are shown in Fig. 3 (c). These outline a strongly swirling DPI flow which acts on the drug particles, forcing them into a spiralling trajectory towards the periphery, where they undergo de-agglomeration due to action of fluid forces and particle collisions. The dispersed flow leaving the mouthpiece spreads laterally from the edges and has a central reverse flow region, which is identified as vortex breakdown. The deagglomerated particles conform to these flow features and cause significant drug losses due to impaction in a patient's mouth-throat region (Fletcher et al., 2021). The aforementioned results unravel the DPI flow characteristics, and thus provide valuable insight into aerosol generation from these inhalers - the knowledge of which has been limited until now.

\footnotetext{
Acknowledgments: The research was supported by the Australian Research Council. The research also benefited from computational resources provided through the NCMAS, supported by the Australian Government, and the Multi-modal Australian ScienceS Imaging and Visualisation Environment (MASSIVE) at Monash University.
}

\section{References}

dos Reis LG, Chaugule V, Fletcher DF, Young PM, Traini D, and Soria J (2021) In-vitro and particle image velocimetry studies of dry powder inhalers. International Journal of Pharmaceutics 592:119966

Fletcher DF, Chaugule V, dos Reis LG, Young PM, Traini D, and Soria J (2021) On the Use of Computational Fluid Dynamics (CFD) Modelling to Design Improved Dry Powder Inhalers. Pharmaceutical Research 38:277-288

Soria J (1996) An investigation of the near wake of a circular cylinder using a video-based digital crosscorrelation particle image velocimetry technique. Experimental Thermal and Fluid Science 12:221-233 